\title{
Financiación, gobernanza y derecho del I+D+i de la Unión Europea: estrategias para la recuperación económica*
}

\author{
Finance, Governance and Law of the R \& $D$ of the \\ European Union: Strategies for Economic Recovery
}

\section{Elena F. Pérez Carrillo**}

SUMARIO: I. Introducción: panorama actual y algunas precisiones conceptuales. II. Fundamentos sobre la relevancia del I+D+i para la reforma del mercado y el modelo productivo europeos. III. Instrumentos jurídicos para promover la investigación aplicada a la innovación $(I+D+i)$. IV. Gobernanza del I+D+i en la Unión Europea. V. Reflexiones, a modo de conclusión. VI. Principal bibliografía y documentación consultada.

* Artículo recibido el 11 de julio de 2011 y aprobado para publicación el 28 de septiembre de 2011.

** Doctora en Derecho, Centro de Estudios y Documentación Europeos, Universidad de Santiago de Compostela, e mail: Elena.carrillo@usc.es.Trabajo redactado al amparo del proyecto de investigación, GC e RSE: respostas xurídicas á crise económica e financeiraINCITE09 202197 PR 02/12/2009 a 01/12/2011 de la Xunta de Galicia. 
RESUMEN: La innovación y la economía basada en el conocimiento, junto con las nuevas tecnologías que sirven de base para adaptar el modelo industrial europeo al siglo actual, se sitúan en el núcleo de las grandes declaraciones programáticas europeas que pretenden orientar la estrategia de la UE en las próximas décadas. Van unidas a renovados esfuerzos por perfeccionar los mecanismos jurídicos que instrumentan la financiación y la gobernanza de la investigación, el desarrollo y la innovación en la Unión Europea.

Palabras clave: $\mathrm{I}+\mathrm{D}+\mathrm{i}$, estrategia europea de recuperación económica, economía basada en el conocimiento, investigación y competitividad

ABSTRACT: Innovation and knowledge-based economy together with new technologies is a base to adapt the European industrial model to the current century. They form the core of major European policy statements intended to serve as a guide for the EU's activities in the coming decades. Such foundations are coupled with renewed efforts to improve funding and governance legal mechanisms of Research, Development and Innovation in the EU.

Descriptors: R\&D, European Strategy for recovery, Knowledge based economy, research, competitiveness

RÉSUMÉ: L'innovation et l'économie basée sur la connaissance, ensemble avec des nouvelles technologies utiles pour adapter le modèle industriel européen au siècle en cours, sont au coeur des grands déclarations programmes européennes qui ont l'intentions d'orienter la stratégie de l'UE en décennies à venir. Sont liés à des efforts renouvelés pour perfectionner les mécanismes juridiques qui mettent en oeuvre le financement et la gouvernance de la recherche, le développement et l'innovation dans l'UE.

Mots-clés: $R+D+i$, la stratégie européenne de relance économique, l'économie basée sur la connaissance, la recherche et la compétitivité. 


\section{INTRODUCCIÓN: PANORAMA ACTUAL

La desaceleración de la actividad está afectando a la actividad económica en todo el mundo, dañando a las familias, trabajadores y empresas, poniendo en tela de juicio el modelo de desarrollo económico y social basado en una sociedad de servicios e intercambios que tiene su origen lejano en el siglo XIX y su antecedente próximo en la posguerra mundial.

En Europa, esta crisis ha sacado a flote una problemática profunda que se había iniciado con anterioridad a 2008: ${ }^{1}$ déficits públicos y privados, recurso excesivo a la financiación externa de la actividad económica y de los propios Estados, desempleo y otros indicios de un modelo que ha devenido poco sostenible. Dentro de la búsqueda de soluciones, desde las instituciones comunitarias se apunta a la necesidad de renovar algunas de las grandes estrategias políticas comunitarias, que a su vez servirán para reformar el mercado y el tejido productivo de la Unión Europea (UE). ${ }^{2}$ Concretamente, se han hecho públicos comunicaciones institucionales y estudios que van marcando las pautas sobre el camino para salir de la crisis y fortalecer el progreso. En estas páginas nos vamos a fijar en algunos de estos textos de reflexión estratégica, cuyos contenidos giran en torno a la necesidad de mejorar la calidad de la investigación en la UE, y de fomentar su aplicación a la industria. Además, nos detendremos en los principales instrumentos jurídicos comunitarios para proteger, financiar y estructurar esta idea, llevándola a la práctica.

En este trabajo aludimos repetidamente a la investigación, desarrollo e innovación $(I+D+i)$. Por ello, conviene comenzar nuestra exposición por clarificar el sentido en el que manejamos dicha terminología. Según el Diccionario de la Real Academia de la Lengua Española, "investigación" tiene como primera acepción, "acción y efecto de investigar", y

1 Pérez Carrillo, E. F., “Europa 2008: retos estratégicos y reforma”, Boletín Mexicano de Derecho Comparado, México, 2008, pp. 279-314. También, más brevemente aludíamos a estas circunstancias en "La crisis de la Bella durmiente", artículo de opinión publicado en la Voz de Galicia, sección Tribuna el 6 de julio de 2011, http: / / avoz.com/opinion/2011/07/06/0003_ 201107G6P15992.htm.

2 Lüder, T., Legislative and Policy Developments in the European Union, Fordham, April 2005. 
se acompaña de la entrada "investigación básica" definida como aquella "que tiene por fin ampliar el conocimiento científico, sin perseguir, en principio, ninguna aplicación práctica”. El concepto de investigación tiene su origen en la epistemología, o ámbito de la filosofía que se dedica al estudio del conocimiento y se representa gráficamente con una "I", dentro de la fórmula "I+D+i". En cuanto al desarrollo - en el sentido que se empleará aquí- es un vocablo fundamentalmente económico y se representa con una "D". La innovación se relaciona con la tecnología como conjunto de conocimientos técnicos ordenados científicamente, que permiten diseñar y crear bienes y servicios, que facilitan la adaptación al medio y satisfacer necesidades. El trinomio "Investigación (I), Desarrollo (D) e innovación (i)” (I+D+i) refleja la relación entre ciencia básica y ciencia aplicada a la industria, al comercio, a los servicios, etc. En política legislativa, se emplea la fórmula "I+D+i" para aludir a como la "investigación básica" (I) sirve para obtener nuevos procesos, productos o servicios mediante el "desarrollo" (D), que a su vez pueden adaptarse para nuevas "aplicaciones" (i).

Investigación, desarrollo e innovación $(\mathrm{I}+\mathrm{D}+\mathrm{i})$ constituyen términos cuyo significado inicial es extra jurídico. Sin embargo, el derecho en tanto que es el orden normativo e institucional que regula las relaciones humanas sobre criterios de justicia, juega un importante papel en su articulación: las normas jurídicas ordenan los procedimientos de asignación de recursos para el $\mathrm{I}+\mathrm{D}+\mathrm{i}$, establecen mecanismos de protección de sus resultados, crean instituciones y organismos para su dirección y gestión dotándolos de procedimientos para su funcionamiento, etcétera.

Distintos documentos estratégicos de la UE sitúan el $\mathrm{I}+\mathrm{D}+\mathrm{i}$ como un pilar fundamental para superar la crisis actual y sentar las bases de un crecimiento a medio plazo; señalando sucintamente que es preciso obtener nuevos conocimientos (I), aplicarlos en la obtención de bienes, productos y servicios cuya entrada en el mercado sirva para satisfacer necesidades (D), y proyectar su valor añadido en campos distintos (i). Concretamente, la necesidad de mejorar la investigación y la innovación están en la base de la Comunicación de la Comisión "Europa 2020: "Una estrategia para un crecimiento inteligente, sostenible e integrador",

3 "Europa 2020: Una estrategia para un crecimiento inteligente, sostenible e integrador" COM (2010) 2020. Adelantábamos un análisis de este texto y de los estudios en los que se 
que es un texto central en la planificación de la Unión Europea para salir de la crisis.

La Estrategia Europa 2020 abarca aspectos distintos (economía, agricultura, industria, etcétera) y entre sus principales conclusiones destaca que el trinomio $\mathrm{I}+\mathrm{D}+\mathrm{i}$ constituye un motor fundamental para la prosperidad social y económica. Propone líneas de acción ejecutiva (“iniciativas emblemáticas”), cada una de las cuales dará lugar a propuestas que a través del derecho legislado y mediante la acción administrativa de la Comisión Europea y del conjunto de los 27 Estados miembros de la UE, la llevarán a la práctica. La iniciativa emblemática "Unión por la innovación" " es otro texto central, desarrollado en el marco de la Estrategia Europa 2020, que aboga por fortalecer la investigación y la innovación de forma que se constituyan en motores del desarrollo industrial y social europeos en el camino hacia la recuperación sostenida. También el proyecto Europa 2030, recientemente hecho público por el Grupo de Reflexión sobre el Futuro de Europa incide con fuerza en la necesidad de vincular investigación, resultados e innovación aplicada a la industria. ${ }^{5}$

El fundamento jurídico de las reformas que se están programando en estos momentos bajo el paraguas de la Estrategia Europa 2020 y de la iniciativa "Unión por la Innovación”, y que reciben la inspiración del Proyecto Europa 2030 se encuentra en el Tratado de Lisboa. El artículo 3o. del Tratado de la Unión Europea (TUE) reformado en Lisboa y que entró en vigor en 2009 reza: "la Unión establecerá un mercado interior. Obrará en pro del desarrollo sostenible de Europa basado en un crecimiento económico equilibrado... Asimismo promoverá el progreso científico y técnico”. El artículo 4.3 del TFUE añade que

en los ámbitos de investigación, desarrollo tecnológico... la Unión dispondrá de competencia para llevar a cabo acciones en particular destinadas a definir y rea-

fundamenta en Pérez Carrillo, E. F., “Europa 2008: retos estratégicos y reforma”, Boletín Mexicano de Derecho Comparado, México, 2008, pp 279-314.

4 Comunicación de la Comisión Europea, "Iniciativa emblemática de Europa 2020 Unión por la innovación” COM (2010) 546.

5 Grupo de Reflexión sobre el Futuro de la UE, Proyecto Europa 2030, Retos y oportunidades, Informe al Consejo Europeo del Grupo de Reflexión sobre el futuro de la UE en 2030, Luxemburgo, 2010. 
lizar programas, sin que el ejercicio de esta competencia pueda tener por efecto impedir a los Estados miembros ejercer la suya.

Conforme a lo dispuesto en los títulos XVII "Industria” y XIX "Investigación y desarrollo tecnológico y espacio” delTFUE; así como en elTítulo II, capítulo 1 "Desarrollo de la investigación" del Tratado Euratom, la UE y sus Estados deben asegurar las condiciones para la competitividad de la industria, y acelerar su adaptación a los cambios estructurales, fomentar la iniciativa y el desarrollo de las empresas (particularmente de las pequeñas y medianas), impulsar la cooperación entre empresas y entre estas y el sector público, etcétera, así como (artículo 173.1, apartado 4 TFUE) "favorecer el aprovechamiento del potencial industrial derivado de las políticas de innovación, de investigación y de desarrollo tecnológico". El artículo 179.1 del TFUE es muy explícito en cuanto a que es objetivo de la UE

fortalecer sus bases científicas y tecnológicas mediante la realización de un espacio europeo de investigación en el que los investigadores, los conocimientos científicos y las tecnologías circulen libremente...

En desarrollo de los citados preceptos de los Tratados, y al amparo de las estrategias contenidas en los documentos mencionados, el legislador comunitario ha creado normas de apoyo financiero y organizativo del $\mathrm{I}+\mathrm{D}+\mathrm{i}$, y está introduciendo reformas para mejorar la capacidad de la investigación aplicada europea como motor de cambio estructural.

\section{FUndAMENTOS SOBRE LA RELEVANCIA DEL I+D+I PARA LA REFORMA DEL MERCADO Y EL MODELO PRODUCTIVO EUROPEOS}

Durante décadas, el mercado interior comunitario se fundamentó en el aprovechamiento de las economías de escala sobre un modelo de capitalismo industrial de producción en masa basado en la mano de obra poco cualificada, que permitió a las clases medias devenir consumidores. Sin embargo, el capitalismo actual presenta unos rasgos bastante distintos 
basados en la diferenciación de productos, la innovación tecnológica, la mano de obra cualificada, adaptada y móvil, y en la financiación empresarial a través del mercado. Superada la hegemonía casi absoluta de Estados Unidos sobre la economía mundial, los países europeos van alcanzando una madurez de "crecimiento pausado". Mientras, los conocidos como emergentes y BRICS aportan un 50\% del crecimiento global, y los países en vías de desarrollo — que atesoran grandes reservas naturales de materias primas — son objeto de atención creciente. ${ }^{6}$ Las economías emergentes están abandonando la competencia en costes en favor de estrategias basadas en la innovación. ${ }^{7}$ El aumento del nivel de vida en estos países y sus capacidades crecientes generan nuevas oportunidades de colaboración. Para contribuir en positivo al nuevo paradigma global, Europa debe mejorar su adaptación a la globalización, renovar los conocimientos subyacentes a su modelo productivo y aprovechar al máximo las utilidades industriales derivadas de tales conocimientos. ${ }^{8}$ El derecho tiene una importante función que cumplir en este proceso.

6 Tamames, R., ¿Cuándo y cómo acabará la crisis? Tratactus lógicus económicus, Madrid, Turpial, 2011. Señala el profesor que cuando, en los años sesenta, el PIB de EE. UU. representaba el $40 \%$ de la economía mundial, la coyuntura se centraba en la expansión o declive de ese país, en las tensiones Este-Oeste y la brecha Norte-Sur. En la actualidad, describe una situación de “tres placas tectónicas” en la que Norte América Europa y Japón ralentizan su desarrollo, los emergentes son el motor del crecimiento y los territorios más atrasados como ciertas zonas de América Latina, de Asia meridional y de África están experimentando importantes incrementos de su PIB como resultado de la puesta en valor de sus grandes reservas de recursos naturales.

7 Entre los factores que motivan el cambio, algunos son exógenos (un proceso de globalización cada vez más intenso, la aparición de áreas continentales con fuerte crecimiento, el gran incremento de los precios de la energía y las materias primas, la internacionalización de la innovación, los problemas relacionados con el cambio climático y una presión migratoria frecuentemente incontrolada o incontrolable). Otros son endógenos como el envejecimiento demográfico, la necesidad de protección del medio ambiente, la obsolescencia del aparato productivo y de servicios, la nueva configuración del sector público, el creciente desfase entre los conocimientos nuevos y el capital humano disponible, etc. Ver sobre estos factores Dictamen del Comité Económico y Social Europeo sobre la Comunicación de la Comisión Regiones europeas competitivas gracias a la investigación y la innovación-Una contribución para un mayor crecimiento y más y mejores puestos de trabajo COM(2007) 474 final, DOUE C 211 de 2008.

8 Commission Staff Working Document, European Competitiveness Report 2010 Accompanying document to the Communication from the Commission to the European Parliament, the Council, the European Economic and Social Committee and the Committee of the Regions. An integrated Industrial 
El mercado interior europeo o "espacio sin fronteras interiores" (artículo 26.2 delTFUE) es a los efectos de este trabajo un nombre genérico en el que incluimos desde procesos o intercambios comerciales, hasta el especio físico (territorio de los 27 Estados miembros) donde tienen lugar. Este mercado, que también cuenta con una vertiente exterior relacionada con los flujos con terceros países está fuertemente enraizado en los instrumentos jurídicos. El artículo 4.3, apartado a del TFUE lo ubica dentro de las competencias compartidas de la UE y sus Estados miembros. Los artículos 26 y 27 TFUE conforman el título I de la Tercera parte delTFUE denominado precisamente "Mercado Interior", que se ubica sistemáticamente como primera "Política y acción interna de la UE”. Estos preceptos de derecho primario se hayan desarrollados por normas de derecho secundario europeo y principalmente por directivas, que de conformidad con lo previsto en los artículos 114 y 115 del TFUE, articulan las libertades básicas de libre circulación de capitales, personas, mercancías y servicios.

Puesto que el mercado interior es en buena medida un concepto producto del derecho, es lógico que a la hora de reformarlo, el debate iniciado con la "Estrategia Europa 2020" y la "iniciativa emblemática Unión por la innovación” trascienda aspectos tan importantes como cuáles deban ser los sectores de investigación, desarrollo y aplicación $(\mathrm{I}+\mathrm{D}+\mathrm{i})$ que convenga potenciar desde la UE, etcétera, para alcanzar a cuáles deben ser las reformas jurídicas que contribuyan a adaptar las reglas del modelo productivo y mercantil europeo al capitalismo global.

\section{Investigación e innovación industrial}

En pocos sitios como en Europa se hace tan claramente patente que investigadores e industriales operan de formas distintas. Así por ejemplo, las universidades y el sector empresarial abordan de manera diferente

Policy for the Globalisation Era Putting Competitiveness and Sustainability at Front Stage $\{\operatorname{COM}(2010) 614\}\{\operatorname{SEC}(2010) 1272\}$ SEC(2010) 1276 final, pp 10-14. También Comisión Europea. Comunicación "Del reto a la oportunidad: hacia un marco estratégico común para la financiación de la investigación y la innovación por la UE”, COM(2011) 48 final. 
la producción de conocimientos. Aquellas reivindican su difusión inmediata, mientras que estas fomentan el secreto, al menos en tanto que no esté garantizada su propiedad intelectual. Este conflicto de intereses es responsable del mantenimiento de la distancia entre estos dos sectores y de las dificultades para establecer cooperaciones productivas y eficientes.

En la Estrategia Europa 2020 y la iniciativa emblemática Unión por la Innovación se reconoce que si bien la Unión Europea gusta de flanquear la bandera de la innovación, aún debe perfeccionar el nivel de coherencia entre los objetivos de la ciencia, la tecnología y la innovación y debe cohesionar las fuerzas investigadoras para que sirvan de palanca para cambiar el modelo productivo y la sociedad. Ambos textos apuestan por incrementar el peso específico de la industria manufacturera interna, así como el de la industria de apoyo, como elementos de sostenibilidad del crecimiento en la economía europea y del modelo social de desarrollo, ${ }^{9}$ pero subrayan que para lograrlos es necesario fortalecer la base de conocimientos de la industria: los conocimientos sustentan la innovación. ${ }^{10}$

\section{Desequilibrios sectoriales}

Una efectiva política de innovación exige un buen conocimiento de las capacidades de los distintos sectores productivos.

Ciertas industrias se encuentran entre las más innovadoras de todos los sectores de la UE, jugando un papel clave en la intensificación de la difusión de las innovaciones tecnológicas. ${ }^{11}$ Así por ejemplo, las empresas de los sectores de consultoría y de suministro de software,

9 El número de empleos derivados de la industria manufacturera junto con la de servicios de apoyo supera el $30 \%$ del empleo en los Estados miembros de la UE. Communication from the Commission to the European Parliament, the Council, the European Economic and social Committee of the Regions, "An Integrated Industrial Policy for the Globalisation Era Putting Competitiveness and Sustainability at Centre Stage", $\{\mathrm{SEC}(2010) 1272\},\{\operatorname{SEC}(2010) 1276\}$.

10 Comisión Europea. Comunicación "2020 Una estrategia para un crecimiento inteligente, sostenible e integrador" COM (2010)2020.

11 Comisión Europea, Competitiveness Report 2010 Accompanying document to the Communication "An integrated Industrial Policy for the Globalisation Era Putting Competitiveness 
dentro del sector de servicios, o las industrias de diseño y arquitectura en relación con la aplicación de nuevas tecnologías, contribuyen sustancialmente al proceso de renovación innovadora. En cambio, la crisis financiera y económica ha afectado muy duramente a otros sectores basados en sistemas obsoletos, como el de la comercialización de bienes intermedios, (por ejemplo las piezas y componentes que representan un aproximado del 50\% del comercio internacional). ${ }^{12}$ Para equilibrar estas desigualdades, desde la UE se propone, entre otras medidas, fomentar la colaboración intersectorial, de forma que las empresas puedan proyectar su actividad de negocio más allá de su campo habitual, así como crear redes de colaboración intersectoriales. ${ }^{13}$ La cooperación industrial permite la puesta en común de actividades cuyas perspectivas resulten de utilidad en varios campos económicos. Así, trabajos como la investigación y el desarrollo, el diseño, los métodos de fabricación, la logística, la comercialización y los servicios a los clientes y usuarios facilitan el aprovechamiento de las dinámicas modernizadoras de los sectores más ágiles en beneficio de la globalidad de la industria y del comercio. Estas cooperaciones se basan en modelos de financiación y gobernanza ordenados desde el derecho.

Por otra parte, Europa debe adaptar, desarrollar, mejorar e incorporar al mercado las capacidades de una mano de obra cualificada. ${ }^{14}$ En particular, algunas tecnologías como la biotecnología o la nanotecnolo-

and Sustainability at Front Stage $\{\operatorname{COM}(2010) 614\}\{\operatorname{SEC}(2010) 1272\} \operatorname{SEC}(2010) " 1276$ final, p. 14.

12 Comisión Europea Competitiveness Report 2010 Accompanying document to the Communication "An integrated Industrial Policy for the Globalisation Era Putting Competitiveness and Sustainability at Front Stage $\{\operatorname{COM}(2010) 614\}\{\operatorname{SEC}(2010) 1272\} \operatorname{SEC}(2010) " 1276$ final, pp 14-16. Se interrumpieron algunas de las cadenas de suministro internacionales más establecidas (destacadamente en la industria automotriz), dando lugar a cambios en las corporaciones multinacionales, y en sus estrategias de aprovisionamiento. Si esta tendencia se confirma, puede tener efectos a largo plazo, entre los que cabe prever restricciones en la internacionalización de algunas actividades, y el retraso en la recuperación.

13 Communication from the Commission to the European Parliament, the Council, the European Economic and social Committee of the Regions. "An Integrated Industrial Policy for the Globalisation Era Putting Competitiveness and Sustainability at Centre Stage" $\{\operatorname{SEC}(2010) 1272\},\{\operatorname{SEC}(2010) 1276\}$.

14 Informe Aho, "Crear una Europa innovadora”, informe del grupo de expertos independientes europeos sobre I+D, presidente: señor Esko Aho, enero de 2006. 
gía, los materiales inteligentes, o los sistemas avanzados de producción industrial, que son fundamentales en las industrias, y los servicios del futuro requieren de mano de obra especialmente capacitada. Ello hace esencial mejorar la cualificación del capital humano europeo mejorando la posición competitiva de las universidades, y centros educativos en términos de excelencia, así como atraer a expertos de otros continentes. El derecho también ocupa un papel central en la consecución de estos objetivos programáticos.

\section{INSTRUMENTOS JURÍDICOS PARA PROMOVER LA INVESTIGACIÓN APLICADA A LA INNOVACIÓN $(\mathrm{I}+\mathrm{D}+\mathrm{I})$}

\section{Derecho de la propiedad industrial (PI): reforzar el sistema de patentes}

La propiedad industrial (PI) constituye la rama jurídica directamente encaminada a proteger los derechos de inventores, promotores e inversores en procedimientos, objetos y técnicas innovadoras. Los derechos de PI llegan a contabilizar hasta tres cuartos del valor de las empresas y son objeto de transacciones comerciales cada vez más importantes. ${ }^{15}$

Los Estados europeos adoptan iniciativas para facilitar la transferencia de conocimientos, pero las significativas discrepancias entre sus marcos regulatorios y prácticas nacionales, así como la variedad de normas sobre gestión de la propiedad industrial dificultan la transferencia transnacional de conocimientos. La protección a nivel comunitario de los derechos de propiedad industrial fomentaría la fabricación y distribución transfronterizas de mercancías en la Unión Europea, en cambio, la falta de títulos unitarios (con alguna excepción parcial, por ejemplo en materia de marcas y de diseño) y la inexistencia de un sistema unificado de resolución de litigios encarecen la protección efectiva de las patentes en Europa.

15 Este sector del ordenamiento jurídico suele conocerse con dos términos distintos: derecho industrial y propiedad industrial (o también derecho de la propiedad industrial), Véase, entre otros, a Fernández-Novoa, C. et al., Manual de la propiedad industrial, Madrid, Barcelona, Buenos Aires, 2009, pp. 47 y ss. sobre la cuestión terminológica, y en general para una análisis detallado de la disciplina. 
Dentro de los derechos de PI destaca por su valor añadido el sistema de patentes, pues bien, Europa no cuenta con una patente comunitaria única sino con dos vías coexistentes: los sistemas nacionales de patentes y el sistema europeo de patentes gestionado por la Oficina Europea de Patentes de Múnich (OEP), ${ }^{16}$ ninguno de los cuales es un instrumento jurídico propiamente comunitario. ${ }^{17} \mathrm{~A}$ falta de avances en este terreno, los costes patentar en Europa siguen siendo desproporcionados, como lo son los derivados de la resolución de litigios, circunstancia que no favorece la recuperación. ${ }^{18}$

Dada la dificultad para alcanzar una patente comunitaria propiamente dicha, ${ }^{19}$ se trabaja sobre medidas complementarias, que por la vía del soft law, es decir, del "derecho blando" o "no obligatorio" puedan aplicarse para mejorar las transferencias de tecnología y conocimientos entre las empresas europeas y entre éstas y los centros de investigación. ${ }^{20}$ En esta línea, una reciente Recomendación de la Comisión establece los

16 La patente nacional fue armonizada mediante la firma de varios convenios internacionales, incluido el Convenio sobre Concesión de Patentes Europeas (Convenio de Múnich) en 1973, al que se han adherido todos los Estados miembros de la UE. En el Convenio de Múnich se establece un procedimiento único de concesión de patente europea. Mediante este Convenio se creó la Oficina Europea de Patentes (OEP) que concede una patente que se convierte inmediatamente en patente nacional y queda sujeta a la las normas nacionales

17 El CPE fue firmado en Múnich en 1973 y en la actualidad forman parte de él 36 países, entre ellos todos los Estados miembros de la UE. Con el Convenio de Múnich sobre la Patente Europea (CPE) de 1973 se prevé un mecanismo centralizado para la tramitación de patentes: la Oficina Europea de Patentes de Múnich (OEP) que gestiona un procedimiento único para la concesión. Sin embargo, una vez concedida, la patente europea está sujeta a las normas nacionales de los Estados contratantes de la OEP designados en la solicitud.

18 La UE está atrasada en relación con EE. UU. y Japón en términos de actividad de patentes. Incluso en Europa, EE. UU. y Japón patentan más que la UE: en la OEP, 137 patentes por millón de habitantes proceden de la UE, frente a 143 patentes de EE. UU. y 174 de Japón. Ello resulta un lastre para la inversión en innovación aplicada. Guedou, "Le système de brevet en Europe”, tresor-eco, núm. 9, enero de 2007, p. 3. También Jaeger, Th., “The EU patent: cui bono et quo vadit?", Common Market Law Review, vol. 47, núm. 1, febrero de 2010, pp. 63-115.

19 La Comunicación de la Comisión al Parlamento Europeo y al Consejo, de 3 de abril de 2007, "Mejorar el sistema de patentes en Europa” (COM (2007) 165 final) recomendó avanzar hacia la patente comunitaria y crear un sistema judicial unificado con competencias sobre los litigios sobre las patentes europeas y las futuras patentes comunitarias, pero hasta el momento esa iniciativa no cuenta con resultados concretos.

20 William, C. et al., Intellectual property: patents, copyright, trademarks and allied rights, 7a. ed., Londres, Sweet \& Maxwell, 2010. También England, P.; “Towards a single, pan-European 
principios que los Estados miembros deberían respetar en la elaboración de las orientaciones o disposiciones sobre transferencia de conocimientos y de gestión de la PI. ${ }^{21}$ Va acompañada de un "Código de buenas prácticas" dirigido a las universidades y a otros organismos públicos de investigación, en el que se fijan aspectos operativos fundamentales para favorecer la explotación y la difusión de los resultados de la investigación financiada con fondos públicos. El "Código" insta (apartado 1) a que los Estados fomenten la investigación aplicada como instrumento estratégico de desarrollo, y a que (apartado 3) apoyen tanto la transferencia de conocimientos por parte de los organismos públicos de investigación, como la iniciativa empresarial para la investigación. ${ }^{22}$

\section{Medidas para la formación y atracción de capital humano altamente cualificado}

Una nueva base productiva exigirá personal capacitado para idearla, y desarrollarla. Ya antes de hacerse evidente la actual crisis económica, una Resolución del Parlamento Europeo de 2005 propuso entre otras medidas: (apartado 1) imprimir una dirección estratégica en los sistemas de educación superior para adaptarlos a las necesidades de la sociedad; (apartado 3) atraer hacia la educación superior a personas procedentes de los medios menos favorecidos. El objetivo último sería mejorar las cualificaciones profesionales en Europa. ${ }^{23}$

standard: common concepts in UK and “continental European” patent law”, European Intellectual Property Review, vol. 32, núm. 6, 2010, p. 259-267.

21 Recomendación de la Comisión del 10 de abril de 2008 sobre la gestión de la propiedad intelectual en las actividades de transferencia de conocimientos y Código de buenas prácticas para las universidades y otros organismos públicos de investigación [C(2008) 1329 final].

22 De cara a fomentar la participación privada en la inversión exigida por la investigación, es esencial contar con instrumentos contables sobre los intangibles (como los derechos de propiedad intelectual) es por ello que el Consejo de la Unión Europea acaba de solicitar que se inicien trabajos prelegislativos al respecto. Véase las Conclusiones del Consejo Europeo, febrero de 2011.

23 Resolución del Consejo y de los representantes de los gobiernos de los Estados miembros reunidos en el seno del Consejo relativa a la movilización del capital intelectual de Europa: crear las condiciones necesarias para que las universidades puedan contribuir plenamente a la estrategia de Lisboa, DO C 292 de 2005. Esta Resolución, instrumento jurídico no vin- 
El Parlamento Europeo ha vuelto a poner de manifiesto en su resolución del 20 de mayo de $2010^{24}$ (numeral 10) la necesidad de introducir mejoras en el funcionamiento de las universidades europeas reforzando la relación "investigación-educación-innovación” y teniendo en cuenta la necesidad de reforzar los vínculos entre universidad y empresa. Propugna también por orientar la inversión de la UE hacia la formación y la investigación, y mejorar la coordinación entre políticas regionales, estatales y comunitarias con fines de investigación aplicada. ${ }^{25}$ En un sentido similar se han manifestado los expertos del Grupo Europa 2030. ${ }^{26}$

Junto con la formación de los europeos, es esencial atraer al capital humano especializado formado en otras latitudes. La Directiva $2005 / 71 / \mathrm{CE}^{27}$ ya establecía unas condiciones especiales que facilitan el

culante o de soft law afecta a aspectos relativos a la reforma educativa que es responsabilidad de los Estados, gozando la UE únicamente de competencia para "llevar a cabo acciones con el fin de apoyar, coordinar o complementar las acciones de los Estados" (artículo 6o., apartado e, TFUE). Dadas las limitaciones competenciales es difícil profundizar en esta línea a través de derecho comunitario imperativo.

24 Resolución del Parlamento Europeo, del 20 de mayo de 2010, sobre el diálogo entre las universidades y las empresas: una nueva asociación para la modernización de las universidades europeas DOC $161 \mathrm{E}$, de 2011. En su numeral 10 pide mejoras en el funcionamiento de las universidades europeas para favorecer la investigación aplicada y reforzar los vínculos entre universidad y empresa. Damos por reproducido el comentario de la nota anterior, en relación con el reparto de competencias en la UE.

25 Las políticas nacionales europeas y regionales contribuyen a superar los fallos del mercado y los ámbitos donde resulta difícil obtener financiación, a crear lazos entre empresas e instituciones dedicadas a la investigación. Pero un sistema eficiente no puede descansar únicamente en la ayuda, sino que ha de fomentar la competencia entre iniciativas para fortalecer a las que presentan mayor interés innovador. Comisión Europea, Comunicación "An Integrated Industrial Policy for the Globalisation Era Putting Competitiveness and Sustainability at Centre Stage"(SEC(2010) 1272), (SEC(2010) 1276). En el mismo sentido, y con propuestas específicas está la Resolución del Parlamento Europeo, del 20 de mayo de 2010, sobre la aplicación de las sinergias entre los fondos asignados a la investigación y la innovación en el Reglamento (CE) no 1080/2006 relativo al Fondo Europeo de Desarrollo Regional y en el Séptimo Programa Marco de Investigación y Desarrollo, en las ciudades y regiones, así como en los Estados miembros y en la Unión (2009/2243(INI), DOC 161E

26 Proyecto Europa 2030, Retos y oportunidades, Informe al Consejo Europeo del Grupo de Reflexión sobre el futuro de la UE en 2030. Luxemburgo 2010.

27 Directiva 2005/71/CE del Consejo, de 12 de octubre de 2005, relativa a un procedimiento específico de admisión de nacionales de terceros países a efectos de investigación científica (DOL 289, 2005). España fue objeto de una condena por retrasar la incorporación al ordenamiento interno de esta directiva(Sentencia del Tribunal de Justicia (Sala Séptima) 
ingreso de investigadores a través de permisos específicos de larga duración con posibilidad de permanencia, situación que también contempla la Directiva 2009/50/CE del Consejo, ${ }^{28}$ actualmente en proceso de reforma para facilitar no sólo la obtención de permisos de entrada por parte de científicos y tecnólogos, sino también su permanencia en unas condiciones de vida atractivas. ${ }^{29}$ Incluso, la normativa sobre seguridad en la libre circulación de personas del espacio Schengen, como el Reglamento (UE) 265/2010 30 crea condiciones especialmente favorables para la entrada y permanencia en Europa de investigadores, científicos y personal altamente cualificado.

\section{Instrumentos y decisiones para la financiación del $I+D$}

El acceso a financiación suficiente es una condición sine qua non para la investigación aplicada al desarrollo, a la innovación y a la industria $(+\mathrm{D}+\mathrm{i})$.

Los programas de la UE para financiar la investigación básica (I) y aplicada (D) son administrados en su mayor parte por los Estados miembros, circunstancia que dificulta la explotación de sinergias. A continuación se reseñan algunas de las actuales vías de financiación comunitaria

de 11 de febrero de 2010-Comisión Europea contra Reino de España (Asunto C-523/08) (publicada en DO, C, de 2009)

28 Directiva 2009/50/CE del Consejo, de 25 de mayo de 2009, relativa a las condiciones de entrada y residencia para empleo altamente cualificado (DOL 155 de 2009).

29 Los informes que sustentan la reforma sugieren la necesidad de incentivos para las profesiones intelectuales y altamente cualificadas como directivos, investigadores o profesionales de la industria; así como crear parques tecnológicos e industriales, plataformas tecnológicas y agrupaciones de empresas orientadas a la investigación aplicada y la transferencia. Véase Informe Aho, "Crear una Europa innovadora", informe del grupo de expertos independientes europeos sobre I+D, Presidente: Sr. Esko Aho, enero de 2006.

30 Reglamento (UE) núm. 265/2010 del Parlamento Europeo y del Consejo, del 25 de marzo de 2010 , por el que se modifica el Convenio de aplicación del Acuerdo de Schengen y el Reglamento (CE) núm. 562/2006 por lo que se refiere a la circulación de personas con visados de larga duración (DOL 085, 2010), cuyo preámbulo estipula que (8.- El presente Reglamento no tiene por objetivo desalentar a los Estados miembros para que no expidan permisos de residencia y no debe afectar a la obligación de los Estados miembros de expedir permisos de residencia para ciertas categorías de nacionales de un tercer país tal como se establece en otros instrumentos de la Unión, en especial, la Directiva 2005/71/CE). 
de la investigación, así como de las reformas jurídicas que se están proyectando e introduciendo para adaptarlas a la Estrategia Europa 2020.

\section{A. Programa marco de $I+D(7$ PM)}

El Séptimo Programa Marco de la Comunidad Europea ${ }^{31}$ (7 PM) fue aprobado en 2006 mediante la decisión 1982/2006/CE del Parlamento y del Consejo. Está dotado con un presupuesto de 53300 millones de euros para apoyar la investigación, el desarrollo tecnológico y de demostración en toda la UE. Es actualmente el instrumento europeo de financiación del $\mathrm{I}+\mathrm{D}+\mathrm{i}$ más potente.

Las acciones financiadas por el 7 PM se ordenan en torno a cuatro programas específicos que se contemplan en su artículo 2o.: cooperación, ${ }^{32}$ ideas, ${ }^{33}$ personas ${ }^{34}$ y capacidades. ${ }^{35}$ Además, financia el Centro Común de Investigación (CCI). ${ }^{36}$

El artículo 7o. de la decisión 1982/CE/2006 preveía una evaluación y revisión del 7 PM en 2010. Esta evaluación provisional ha confirmado su papel fundamental para la creación de las redes europeas de investigación, y otras infraestructuras. ${ }^{37}$ Demostró además, el éxito de dos

31 Decisión núm. 1982/2006/Ce del Parlamento Europeo y del Consejo del 18 de diciembre de 2006 relativa al Séptimo Programa Marco de la Comunidad Europea para acciones de investigación, desarrollo tecnológico y demostración (2007 a 2013) (DOL 412, 2006)

32 Dentro de este subprograma se financia la investigación en cuestiones como la salud, las nanotecnologías la energía el transporte, el medio ambiente (artículo 2o., i) .

33 Apoyo a la investigación "impulsada por los investigadores realizada en todos los campos por equipos nacionales o transnacionales individuales que compitan a nivel europeo” (artículo 2o., ii).

34 Fortalecimiento, cuantitativo y cualitativo, del potencial humano de la investigación y del desarrollo tecnológico en Europa, así como el estímulo de la movilidad (artículo 2o., iii)

35 Apoyo a aspectos clave de la capacidad de investigación e innovación europea, como las infraestructuras de investigación; las agrupaciones regionales impulsadas por la investigación; el desarrollo de todo el potencial investigador de las regiones comunitarias de convergencia y ultraperiféricas; investigación destinada a las pequeñas y medianas empresas (PYME) las cuestiones de "Ciencia y sociedad"; apoyo al desarrollo coherente de las políticas; actividades "horizontales" de cooperación internacional (artículo 2o. iv).

36 El CCI es un servicio de la Comisión que presta apoyo científico y técnico a la elaboración y aplicación de las políticas de la UE. Originalmente se creó en el marco de la Comunidad Europea para la Energía Atómica, Euratom véase infra.

37 La Decisión núm. 1982/2006/CE del Parlamento Europeo y del Consejo, del 18 de diciembre de 2006, relativa al Séptimo Programa Marco de la Comunidad Europea para accio- 
instrumentos creados al amparo del 7 PM que se integran respectivamente en la gobernanza del $\mathrm{I}+\mathrm{D}+\mathrm{i}$ europeo y en sus mecanismos financieros, y a los que nos referiremos más adelante: el Consejo Europeo de Investigación $(\mathrm{CEI})^{38}$ y el Instrumento de Financiación de Riesgos Compartidos (IFRC). ${ }^{39}$ Ha evidenciado, por otra parte, ciertos aspectos que deben mejorar como la vinculación entre la investigación y la innovación, y el fortalecimiento de la relación entre competitividad y objetivos sociales. ${ }^{40}$ Adicionalmente, propugna una simplificación en las normas de solicitud y de gestión de las ayudas. ${ }^{41}$

\section{B. Programa marco de innovación y competitividad (PIC)}

Este programa de financiación fue puesto en marcha a través de la Decisión legislativa del Parlamento Europeo y del Consejo (1639/2006/ CE) ${ }^{42}$ con arreglo al apartado 37 del Acuerdo interinstitucional de 17

nes de investigación, desarrollo tecnológico y demostración (2007 a 2013) (7 PM) contiene en su artículo 7, apartado 2, la base jurídica para la realización de una evaluación intermedia del $7^{\circ}$ PM (DO L 412 de 30.12.2006)

38 El Consejo Europeo de Investigación (CEI) es un medio de ejecución del Programa Específico "Ideas", véase infra.

39 La Decisión núm. 1982/2006/CE (DO L 412 de 30.12.2006) y las correspondientes a sus programas específicos “Cooperación” (Decisión 2006/971 / CE del Consejo, del 19 de diciembre de 2006) y “Capacidades” (Decisión 2006/974/CE del Consejo, del 19 de diciembre de 2006) exigían que expertos independientes llevaran a cabo una evaluación de la aplicación del Instrumento de Financiación del Riesgo Compartido (IFRC). Esta evaluación puede consultarse en http: / / ec.europa.eu/research/evaluations. vid infra

40 Evaluación intermedia 7 PM, http://ec.europa.eu/research/evaluations/index_en.cfm?pg= fp 7 .

${ }^{41}$ Además de los resultados de la evaluación intermedia, la simplificación se promueve desde otros foros. Así, el Parlamento ha pedido a la Comisión que simplifique la burocracia para el 7 PM (y el PIC), incluyendo la presentación de solicitudes para los diferentes programas de apoyo mediante programas informáticos con manuales normalizados, Resolución del Parlamento Europeo, de 20 de mayo de 2010, sobre la aplicación de las sinergias entre los fondos asignados a la investigación y la innovación en el Reglamento (CE) núm. 1080/2006 relativo al Fondo Europeo de Desarrollo Regional y en el Séptimo Programa Marco de Investigación y Desarrollo, en las ciudades y regiones, así como en los Estados miembros y en la Unión (2009/2243(INI), DOC 161E.

42 DECISIÓN No 1639/2006/CE del Parlamento Europeo y del Consejo del 24 de octubre de 2006 por la que se establece un programa marco para la innovación y la competitividad (2007-2013) (DOL 310 de 2010). 
de mayo de 2006 entre el Parlamento Europeo, el Consejo y la Comisión sobre disciplina presupuestaria y buena gestión financiera. ${ }^{43}$ Según el artículo 3o. de esta Decisión, el PIC dispone de un presupuesto de 3600 millones de euros y su propósito es fomentar la competitividad de la industria europea, constituyendo - de conformidad con su artículo 2o._ las PYME su objetivo principal.

El PIC promueve el acceso a la financiación de la PYME (artículo 1.1-a) y respalda las políticas de apoyo a la innovación (artículos 1.1-b, 13, 14 y 15), financia servicios transnacionales de apoyo a las empresas y a la innovación (artículos 1.2.a, y 11). Contribuye al desarrollo de la sociedad de la información estimulando la aceptación y el uso de las tecnologías de la Información y la Comunicación (TIC); fomenta la utilización de energías renovables y las medidas de eficiencia energética. De conformidad con el artículo 16 de la decisión 1639/2006/CE, el PIC da prioridad al desarrollo de estudios sobre la reforma económica y administrativa vinculada a la innovación.

Ha sido objeto de revisión intermedia, ${ }^{44}$ ejercicio que ha evidenciado su importante papel como apoyo financiero a las PYME y a su integración en redes (Enterprise Europe Network), como impulsor de la innovación ecológica y de proyectos para la innovación en las TIC.

\section{Instrumento de Financiación de Riesgo Compartido (IFRC)}

El IFRC se creó a raíz de una petición del Consejo Europeo de diciembre de 2005 dentro de las acciones que se estaban entonces articulando en torno al proyecto de 7 PM, y gracias a la colaboración entre la Comisión Europea y el Banco Europeo de Inversiones (BEI) que en conjunto aportaron 2 billones de euros. Su objetivo es cubrir las posibles pérdidas en los créditos facilitados por el BEI y posibilitar créditos situados en torno a los 10000 millones EUR para las actividades de $\mathrm{I}+\mathrm{D}+\mathrm{i}$ a cargo de empresas privadas o instituciones públicas. De conformidad con el

43 DO C 139 de 2006.

${ }^{44}$ Ver evaluación intermedia del PIC en: http://ec.europa.eu/cip/files/docs/interim_evalua tion_report_march2010_en.pdf. 
anexo II del Reglamento 1982/2006/CE del 7 PM, ${ }^{45}$ la contribución financiera de la UE al IFRC se desglosa en dos partes: un primer tramo de 500 millones EUR para el periodo 2007-2010 y un segundo tramo de otros 500 millones para el periodo 2011-2013, previa realización de una evaluación intermedia.

La evaluación intermedia del 7 PM ha evidenciado el éxito del IFRC, ${ }^{46}$ dando lugar a la apertura del segundo tramo de inversión en pleno proceso de crisis y recuperación económica.

EL IFRC financia operaciones con alto perfil de riesgo que abarcan la investigación de base y aplicada, el desarrollo tecnológico, y la investigación aplicada. Sus bases reguladoras permiten intervenir en multitud de proyectos (que superen los requisitos de viabilidad técnica y ambiental del BEI) desde sus fases iniciales de estudios de viabilidad a las de desarrollo industrial; pudiendo financiar inversiones en materiales y equipos, o en inmateriales (coste de explotación de la investigación y desarrollo, gastos de protección a través de derechos de PI), arrendamientos financieros, $\mathrm{y}$ amortizaciones.

\section{Inversión privada en la financiación del $I+D$}

Los documentos estratégicos a los que aludíamos en las primeras páginas de este trabajo indican que la escasa inversión privada en investigación e innovación constituye un notable punto débil en el $\mathrm{I}+\mathrm{D}+\mathrm{i}$ de Europa. Es por ello un reto prioritario el lograr que la inversión en investigación resulte más atractiva para el inversor privado.

La UE se viene planteando desde el año 2000 el desiderátum de invertir el 3\% de su PIB en I+D, y ello ha servido para centrar la atención

45 Decisión núm. 1982/2006/CE del Parlamento Europeo y del Consejo, de 18 de diciembre de 2006, relativa al Séptimo Programa Marco de la Comunidad Europea para acciones de investigación, desarrollo tecnológico y demostración (2007 a 2013) (7 PM).

46 Como decíamos en relación con el 7 PM, la evaluación intermedia de este programa ha evidenciado el éxito del IFRC. La Decisión núm. 1982/2006/CE del 7 PM y las correspondientes a sus programas específicos “Cooperación” (Decisión 2006/971/CE del Consejo, de 19 de diciembre de 2006) y “Capacidades” (Decisión 2006/974/CE del Consejo, de 19 de diciembre de 2006) exigían que expertos independientes llevaran a cabo una evaluación de la aplicación del Instrumento de Financiación del Riesgo Compartido (IFRC). Esta evaluación puede consultarse en http: / / ec.europa.eu/research/evaluations. 
en la necesidad de concitar esfuerzos públicos y privados en torno a un mismo fin, aunque cuantitativamente, tal objetivo no se haya cumplido todavía. ${ }^{47}$ Dado el actual deterioro de las finanzas públicas en los Estados miembros, el Parlamento Europeo ha propuesto explorar nuevas soluciones como programas cofinanciados por la política regional europea y la política agraria común para asegurar el eficaz y eficiente apoyo financiero que permita la coincidencia de la financiación pública y los incentivos privados, ${ }^{48}$ el refuerzo de las sinergias entre fondos de investigación, innovación, política regional y el programa marco de $\mathrm{I}+\mathrm{D}$, a través de un sistema de gobernanza a múltiples niveles (europeo, estatal, regional, local, que incluya la participación financiera privada) que permita la eficaz asignación de fondos. ${ }^{49}$

Con la crisis actual, y la consiguiente contracción del crédito, ${ }^{50}$ determinadas acciones comunitarias como las relativas al fomento del capital riesgo ocupan un papel preponderante en la atracción de capital

47 Como señalábamos más arriba, en sus recientes documentos estratégicos (Estrategia 2020, principalmente), la Comisión propone mantener el horizonte del $3 \%$, complementado con un indicador que refleje la intensidad de la $\mathrm{I}+\mathrm{D}+\mathrm{i}$ por sectores y áreas geográficas. La modernización de la base industrial de Europa así como la actualización de la infraestructura de apoyo sectorial requiere sustanciales inversiones que permitan involucrar no sólo al capital público, sino también al privado. Ello incluye la financiación para la puesta en marcha de empresas, la inversión en empresas en crecimiento así como la financiación de proyectos de $\mathrm{I}+\mathrm{D}$ que a menudo no pueden financiarse a través del capital corriente (cash flow).

48 Véase la exposición de motivos de la Communication from the Commission to the European Parliament, the Council, the European Economic and social Committee of the Regions "An Integrated Industrial Policy for the Globalisation Era Putting Competitiveness and Sustainability at Centre Stage" $\{$ SEC(2010) 1272\}, \{SEC(2010) 1276\}. En esta línea, desde la Instituciones comunitarias se anima a prestar apoyo temporal a las empresas para que inviertan en proyectos innovadores susceptibles de contribuir a mejorar la posición tecnológica de la Comunidad. Comunicación de la Comisión-Marco temporal comunitario aplicable a las medidas de ayuda estatal para facilitar el acceso a la financiación en el actual contexto de crisis económica y financiera. DOC 016 de 2009.

49 Resolución del Parlamento Europeo, de 20 de mayo de 2010, sobre la aplicación de las sinergias entre los fondos asignados a la investigación y la innovación en el Reglamento (CE) no 1080/2006 relativo al Fondo Europeo de Desarrollo Regional y en el Séptimo Programa Marco de Investigación y Desarrollo, en las ciudades y regiones, así como en los Estados miembros y en la Unión (2009/2243(INI), DOC 161E.

50 Ver por todos Muñoz de Bustillo, R. y Bonete Perales, R., Introducción a la Unión Europea: un análisis desde la economía, Madrid, 2009 
privado para financiar acciones de $\mathrm{I}+\mathrm{D}+\mathrm{i} .{ }^{51} \mathrm{El}$ esfuerzo de financiación exige la acción conjunta y coordinada estratégicamente de la UE y de sus Estados miembros. ${ }^{52}$

Actualmente, la obtención de financiación para proyectos de $\mathrm{I}+\mathrm{D}+\mathrm{i}$ por parte de las empresas es difícil, y más aun en el caso de las PYME que pretendan obtenerla con vistas a investigación. Además de fomentar el "espíritu emprendedor" de los ciudadanos, ${ }^{53}$ las instituciones de la UE realizan — a través de sus competencias administrativas - acciones directas y/o de fomento de proyectos que faciliten el acceso privado a recursos económicos. A modo de ejemplo, la Comisión ha establecido un Foro de Financiación de Pequeñas y Medianas Empresas para asesorar y para difundir buenas prácticas y soluciones innovadoras en el acceso a la financiación por parte de las empresas de reducidas dimensiones. ${ }^{54}$

51 La tendencia natural del capital riego a invertir prioritariamente en tiempos de expansión se sitúa en la base de las recomendaciones de algunos organismos europeos como el Comité Económico y Social Europeo, que se ha mostrado favorable a fomentar la inversión privada en empresas en sus primeras fases a través de incentivos fiscales. Véase Dictamen del Comité Económico y Social Europeo (CES) sobre la "Comunicación de la Comisión al Consejo, al Parlamento Europeo, al Comité Económico y Social Europeo y al Comité de las Regiones Eliminación de obstáculos para las inversiones transfronterizas mediante fondos de capital riesgo", INT / 404 Inversiones transfronterizas, Bruselas, 22 de octubre de 2008. El CES pone como ejemplo el programa británico de inversión empresarial (UK Enterprise Investment Scheme). Más detalles en Pérez Carrillo, E. F., “Europa 2008: retos estratégicos y reforma", Boletín Mexicano de Derecho Comparado, Mexico, 2008, pp. 279-314.

52 Es bastante común encontrar debates sobre cómo los diferentes Estados compiten para atraer a los inversores. En este sentido, y aunque Europa pueda percibirse como un espacio de inversión único, la armonización de los mercados industriales y financieros de Europa todavía tiene un largo camino por recorrer, y mientras tanto, las prácticas locales resurgen en particular en épocas de crisis. Las inversiones de capital impulsan el desarrollo económico tanto de forma directa, mediante la financiación de la actividad de producción, como indirectamente, mediante los mercados que permiten el acceso a la financiación a las empresas con mejores resultados. E. Kamar, "Beyond Competition for Incorporations", Georgetown Law Journal, agosto de 2006, pp. 1725-1770, en particular 1730. También en ese mismo sentido Proyecto Europa 2030, Retos y oportunidades, Informe al Consejo Europeo del Grupo de Reflexión sobre el futuro de la UE en 2030. Luxemburgo 2010

53 Tamames, R., ¿Cuándo y cómo acabará la crisis? Tratactus lógicus económicus, Madrid, Turpial, 2011.

54 El Fondo Europeo de Inversiones (FEI) se encarga de su gestión por cuenta de la Comisión e interviene a través de entidades financieras nacionales, regionales y locales (bancos u otras entidades crediticias y fondos de inversión), garantizando así una financiación de proxi- 


\section{E. Simplificación de los programas de financiación de la investigación}

El sistema de apoyo a las actividades de $\mathrm{I}+\mathrm{D}$ se ha hecho muy complejo con multitud de programas nacionales y regionales, de iniciativas intergubernamentales y de procedimientos de financiación. Las abundantes normas y calendarios de la miríada de convocatorias existentes generan una ingente carga administrativa y desalientan la participación, sobre todo en el caso de las PYME, y de la cooperación transfronteriza.

Los principales instrumentos normativos que rigen la relación entre programas de financiación europea de la investigación, y centros dedicados al I+D son el Reglamento (CE) 1906/2006 55 y el Reglamento (Euratom) 1908/2006 ${ }^{56}$ respecto de los que la Comisión Europea acaba de proponer reformas y simplificaciones ${ }^{57}$ con el objetivo de reencontrar el equilibrio entre libertad de acción y control. En su decisión del 28 de febrero de $2011^{58}$ plantea la posibilidad de pasar de una financiación basada en los costes a una financiación basada en los resultados, y en procedimientos simplificados. ${ }^{59}$ Además, la Comisión ha propuesto

midad. Más información en la Web de la Representación de la Comisión Europea en España: http:// ec.europa.eu/spain/novedades/empresas/entrevista-daniel-calleja_es.htm.

55 Reglamento (CE) núm. 1906/2006 del Parlamento Europeo y del Consejo, del 18 de diciembre de 2006, por el que se establecen las normas de participación de empresas, centros de investigación y universidades en las acciones del Séptimo Programa Marco, y las normas de difusión de los resultados de la investigación (2007-2013). DO L 391 de 2006.

56 Reglamento (Euratom) núm. 1908/2006 del Consejo, del 19 de diciembre de 2006, por el que se establecen las normas de participación de empresas, centros de investigación y universidades en las acciones del séptimo programa marco de la Comunidad Europea de la Energía Atómica, y las normas de difusión de los resultados de la investigación (2007-2011) DO L 400 de 2006.

57 Comunicación de la Comisión "Simplificar la ejecución de los programas marco de investigación”, $\operatorname{COM}(2010) 18$.

58 Decisión 2011/161/UE, Euratom de la Comisión, del 28 de febrero de 2011, por la que se modifica la Decisión C (2008) 4617, relativa a las normas para la presentación, evaluación y selección de propuestas y la adjudicación de financiación para acciones indirectas en virtud del Séptimo Programa Marco de la Comunidad Europea de Acciones de Investigación, Desarrollo Tecnológico y Demostración (2007 a 2013) y el Séptimo Programa Marco de la Comunidad Europea de la Energía Atómica (Euratom) de Acciones de Investigación y Formación en Materia Nuclear (2007 a 2011), DO L 75 de 2011.

59 En el mismo sentido véase Dictamen del Comité Económico y Social Europeo sobre la Comunicación de la Comisión al Parlamento Europeo, al Consejo, al Comité Económico y 
una revisión del Reglamento financiero ${ }^{60}$ que de aprobarse permitiría el cálculo de los importes a tanto alzado, el reembolso basado en las prácticas contables del beneficiario, y facilitaría las solicitudes presentadas por asociaciones público-privadas.

\section{GOBERNANZA DEL I+D+I EN LA UNIÓN EUROPEA}

Con este título de "Gobernanza del I+D en la UE" aludimos al conjunto de organismos y estructuras para la dirección y gestión de la investigación, desarrollo e innovación en la UE. Para avanzar en esta gobernanza, el Consejo puso en funcionamiento en mayo de 2008 el llamado "Proceso de Liubliana" ${ }^{61}$ con el que se pretendía adoptar un marco legal institucional para la creación y gestión de organismos y redes de investigación paneuropeos. El Proceso de Liubliana se basaba en la puesta en común de los recursos de investigación de la UE y de sus Estados miembros para compartir la experiencia de asesores altamente especializados en el diseño y evaluación de la política de Investigación europea; para fortalecer y reformar la educación superior; facilitar la creación de redes y lograr mayor cooperación entre universidades, centros de investigación y empresas.

Con la entrada en vigor en 2009 del Tratado de Lisboa que reformó el Derecho Primario europeo, la libre circulación del conocimiento constituye una auténtica "quinta libertad" europea, ${ }^{62}$ reconocida expresamente en el artículo 179.1 del TFUE. Además, el mismo TFUE (artículo 4.3) confiere a la UE competencia para llevar a cabo acciones, así como para definir y realizar programas en el ámbito de la investiga-

Social Europeo y al Comité de las Regiones-Simplificar la ejecución de los programas marco de investigación, COM (2010) 187 final.

60 Comisión Europea, Propuesta de Reglamento del Parlamento Europeo y del Consejo sobre las normas financieras aplicables al presupuesto anual de la Unión COM (2010) 815.

${ }^{61}$ Conclusiones del Consejo Europeo de mayo de 2008 (9076/08). Ver también la Propuesta de Reglamento del Consejo Comisión Europea, "Propuesta de reglamento del Consejo relativo al marco jurídico comunitario para las infraestructuras de investigación europeas (ERI)”, COM (2008) 467.

62 Pérez Carrillo, E. F., “Europa 2008: retos estratégicos y reforma”, Boletín Mexicano de Derecho Comparado, México, 2008, pp. 279-314. 
ción y del desarrollo tecnológico, sin que ello afecte a la propia de los Estados. Sobre estas bases el legislador europeo ha ido dando forma a una serie de organismos y redes de colaboración que constituyen instrumentos de la gobernanza europea del I+D+i. Algunos, de antigua creación se ven fortalecidos en la actualidad. Otros son más recientes. Todos ellos dan visibilidad a la amplia comunidad de $\mathrm{I}+\mathrm{D}+\mathrm{i}$ europea, permiten gestionar los existentes programas de financiación así como fomentar la relación entre investigadores, inversores, académicos e industriales, y facilitan proyectos conjuntos.

A continuación relacionamos algunas de las estructuras que, dentro de la gobernanza europea del $\mathrm{I}+\mathrm{D}+\mathrm{i}$ están cosechando más éxitos.

\section{Centro Común de Investigación (CCI)}

El Centro Común de Investigación (Joint Research Centre, JRC) es una Dirección General de la Comisión Europea que recibe desde hace décadas financiación de los Programas Marco de I+D. En el actual periodo de programación, sus actividades se rigen por la decisión 2006/975/ CE del Consejo, ${ }^{63}$ en el marco del 7 PM.

Consta de siete Institutos de investigación situados en cinco Estados miembros (Bélgica, Alemania, Italia, Holanda y España). Al ser un servicio de la Comisión, es esta institución la que elabora el programa de trabajo plurianual del CCI, así como un Programa específico en el que se precisan detalladamente los objetivos y las prioridades científicas y tecnológicas, y el calendario de ejecución de cada periodo de programación. Estos documentos se redactan teniendo en cuenta las actividades de investigación realizadas por los Estados miembros, otros Estados asociados y las organizaciones europeas e internacionales. Prestar un apoyo científico y técnico impulsado por los clientes al proceso

${ }^{63}$ En el actual periodo de programación, sus actividades se rigen por la Decisión 2006/975/CE del Consejo, de 19 de diciembre de 2006, relativa al Programa Específico que debe ejecutar mediante acciones directas el Centro Común de Investigación dentro del Séptimo Programa Marco de la Comunidad Europea de Acciones de Investigación, Desarrollo Tecnológico y Demostración (2007-2013) (DOL 400 del 30 de diciembre de 2006). Prestar un apoyo científico y técnico impulsado por los clientes al proceso de elaboración de las políticas comunitarias, respaldando la aplicación y el seguimiento de las políticas actuales y respondiendo a las nuevas demandas políticas. 
de elaboración de las políticas comunitarias, respaldando la aplicación y el seguimiento de las políticas actuales y respondiendo a las nuevas demandas políticas.

El CCI proporciona apoyo científico y técnico para la elaboración, desarrollo, aplicación y supervisión de políticas de la Unión Europea. Funciona como centro de referencia en materia científica y tecnológica para la Unión, manteniéndose próximo al proceso de formulación de políticas. Sirve al interés común de los Estados miembros, al tiempo que es independiente de intereses particulares, ya sean privados o nacionales. Una de sus principales funciones es la de promover la transferencia de las tecnologías derivadas de los resultados de la investigación con el fin de crear una plusvalía industrial y de favorecer las políticas comunitarias en el ámbito de la innovación.

En el actual periodo de programación, el CCI ha centrado sus recursos en la necesidad de responder a los retos de la investigación y la tecnología derivados de la necesidad de hacer evolucionar a las políticas europeas, estableciendo sinergias con otros organismos de los Estados miembros, las agencias de la UE y otras instituciones de la UE.

\section{Instituto Europeo de Innovación y Tecnología (EIT)}

En el marco del anteriormente citado Proceso de Liubliana se creó el Instituto Europeo de Innovación y Tecnología con el objetivo primordial de integrar la educación, la investigación y la innovación de los Estados europeos, y de llegar a ser un modelo para la promoción de la innovación abierta y del intercambio de conocimientos entre organismos públicos de investigación y empresas.

Constituido en 2008, tiene su sede social en la ciudad de Budapest, en Hungría. Es un organismo autónomo de la UE que reúne a los sectores de la enseñanza superior, la investigación y la empresa. Estimula la innovación basada en la investigación más puntera. Su funcionamiento, más flexible que otras infraestructuras comunitarias, pretende dotarlo de agilidad de forma que resulte atractivo para el sector empresarial. ${ }^{64}$

${ }^{64}$ Rohrbeck, R, Pirelli, L., The European Institute of Innovation and Technology: How to Steer a Multi-Stakeholder Innovation Ecosystem (18 de abril de 2010). DIME Confe- 
A través de sus comunidades de conocimiento e innovación (CCI) refuerza los vínculos entre la industria, la educación y la comunidad investigadora. Integra una auténtica cadena de innovación que abarca desde la educación y la investigación a la comercialización, y elabora modelos para la reforma del sistema productivo basado en el $\mathrm{I}+\mathrm{D}+\mathrm{i}$. Participa en el proceso de transmisión y difusión de los resultados de la investigación y en su aplicación comercial y empresarial. ${ }^{65}$

\section{Oficina Europea del Espacio Europeo de Investigación (ERAB)}

La ERAB fue creada mediante la decisión 2008/111/CE, Euratom de la Comisión de 2007. ${ }^{66}$ Es un organismo asesor de la Comisión (artículo 1o.) cuyos miembros (artículo 4o.) son escogidos entre expertos científicos y académicos respetándose el equilibrio en cuanto a sus disciplinas de especialización, experiencia, etcétera. Emite dictámenes a petición de la Comisión o por iniciativa propia.

Elabora un informe anual sobre la situación del espacio europeo de investigación que constituye un objetivo de la UE desde la entrada en vigor del Tratado de Lisboa (artículo 179.1 delTFUE). Desde su primer informe anual, hecho público en 2009, viene abogando por la importancia de fomentar un "nuevo renacimiento" de la investigación en Europa, que a su vez suponga un instrumento de superación de la crisis. ${ }^{67}$ ERAB ha definido seis áreas políticas generales en las que la actuación de la UE es necesaria para contribuir a restablecer la confianza entre sociedad y comunidad de investigación: 1) crear un Espacio Europeo de Investigación unificado; 2) dar soluciones a los grandes retos (energético, envejecimiento de la población, adaptación a nuevas tecnologías, etcétera) a través de soluciones novedosas que deriven de investigaciones

rence - Organizing for Networked Innovation, April 2010. Disponible en http://ssrn.com/ abstract $=1591751$.

65 Véase http://eit.europa.eu/.

66 2008/111/CE,Euratom: Decisión de la Comisión, del 7 de diciembre de 2007, por la que se crea la Oficina del Espacio Europeo de Investigación.

${ }^{67}$ ERAB, "Preparar a Europa para un nuevo Renacimiento: una visión estratégica del Espacio Europeo de Investigación”, Bruselas, 2009. 
punteras; 3) interacción entre la ciencia y la sociedad; 4) colaboración entre los sectores público y privado en favor de una innovación abierta; 5) fomento de la excelencia, y 6) promoción de la cohesión (económica y social, territorial, etcétera).

4. Consejo Europeo de Investigación (CEI)

Fue creado en virtud de la decisión de la Comisión 2007/134/CE ${ }^{68}$ como instrumento de la política de investigación comunitaria conforme a las disposiciones del 7 PM. En 2008, la Comisión le delegó la responsabilidad de la ejecución del sub programa "Ideas", (dotado, dentro del 7 PM con 7.510 millones de euros para el periodo 2007-2013) y en julio de 2009, le concedió autonomía administrativa.

Su estructura comprende un consejo científico independiente y una estructura de ejecución especializada en forma de Agencia Ejecutiva de la Comisión. ${ }^{69} \mathrm{~A}$ efectos de revisión de las propuestas que le son sometidas utiliza la evaluación inter pares a través de expertos seleccionados por su Consejo Científico entre los que se encuentran científicos y representantes del sector académico de todas las disciplinas, organizados en tres ámbitos: ciencias físicas e ingeniería, ciencias de la vida, y ciencias sociales y humanidades.

Gestiona fundamentalmente dos tipos ayudas a la investigación: por un lado, las subvenciones para investigadores independientes que inician su carrera, y, por otro, las subvenciones del CEI para investigadores experimentados. A partir de 2011 se abre una nueva vía a través de la cual, los beneficiarios de ayudas del CEI podrán recibir adicionalmente financiación de "demostraciones" de proyectos, para verificar el potencial comercial de su propia investigación aplicada a la innovación. Pres-

68 Decisión de la Comisión 2007/134/CE de 2 de febrero de 2007, por la que se establece el Consejo Europeo de Investigación (DO L 57 de 2007), modificada por la Decisión de la Comisión, de 27 de abril de 2009 , 2009/357/CE y la Decisión de la Comisión, del 12 de enero de 2011 (DO L 9 del 13 de enero de 2011).

69 2008/37/CE: Decisión de la Comisión, de 14 de diciembre de 2007, por la que se crea la Agencia Ejecutiva del Consejo Europeo de Investigación para la gestión del programa específico comunitario Ideas en el campo de la investigación en las fronteras del conocimiento en aplicación del Reglamento (CE) núm. 58/2003 del Consejo (DO L 9 de 2008). 
tar un apoyo científico y técnico impulsado por los clientes al proceso de elaboración de las políticas comunitarias, respaldando la aplicación y el seguimiento de las políticas actuales y respondiendo a las nuevas demandas políticas.

Prestar un apoyo científico y técnico impulsado por los clientes al proceso de elaboración de las políticas comunitarias, respaldando la aplicación y el seguimiento de las políticas actuales y respondiendo a las nuevas demandas políticas.

\section{Consorcios de infraestructuras de investigación (ERIC)}

Los consorcios de infraestructuras de investigación, conocidos por sus siglas en inglés como ERIC, son estructuras constituidas por unidades de investigación situadas en los Estados miembros de la UE, con la posible integración de centros extracomunitarios. El Reglamento (CE) núm. 723/2009 del Consejo ${ }^{70}$ les dota de una base jurídica única.

La Decisión sobre la constitución de un ERIC la adopta la Comisión Europea teniendo en cuenta una evaluación previa, así como la opinión de los Estados miembros de la UE. Una vez aprobada, la decisión se publica en el Diario Oficial de la Unión Europea. Para constituirse, los asociados o entidades afiliadas deben pertenecer al menos a tres Estados miembros, a los que se pueden asociar miembros de los terceros países o de organizaciones intergubernamentales. Una vez constituido el consorcio tendrá personalidad jurídica siéndole aplicable el derecho comunitario, y subsidiariamente el derecho del Estado en que se encuentre la sede social estatutaria o del Estado en que se desarrollen las actividades que afecten a cuestiones técnicas y de seguridad. El ERIC, que está dotado de personalidad jurídica, debe tener su sede en alguno de sus afiliados (Estado miembro o país asociado al 7 PM) y su denominación debe incluir la abreviatura ERIC.

La creación de infraestructuras de investigación al amparo del Reglamento 723/2009 se justifica en requisitos impuestos desde esta norma

70 Reglamento (CE) núm. 723/2009 del Consejo, del 25 de junio de 2009, relativo al marco jurídico comunitario aplicable a los Consorcios de Infraestructuras de Investigación Europeas (ERIC) DO L 206 de 2009. 
de derecho derivado europeo, que incluyen el contribuir a la ejecución de las actividades europeas de investigación; el representar un valor añadido en los campos científicos y tecnológicos a nivel europeo e internacional; el estar abierta a los investigadores de los Estados miembros y de los países asociados al programa marco comunitario I+D; el favorecer la movilidad de los investigadores y el intercambio de conocimientos en el territorio de la UE, así como el contribuir a la mejor difusión y utilización óptima de los resultados de las actividades de investigación.

\section{REFLEXIONES, A MODO DE CONCLUSIÓN}

Investigación, desarrollo e innovación son motores para mejorar la productividad, para mantener el nivel de vida de la población y el liderazgo de los Estados. En la recuperación de la actual crisis, la investigación aplicada está llamada a constituir un elemento fundamental, pues hacer frente a los retos globales de la competitividad y sostenibilidad exige innovación, y ésta se basa en la investigación y el desarrollo.

Existe un amplio consenso sobre la necesidad de desarrollar las condiciones que permitan explotar mejor el potencial comercial de las políticas de investigación, desarrollo e innovación. Sobre esta base, en la UE se está produciendo un debate de calado, que ha cristalizado en documentos como la Estrategia Europa 2020 y a la iniciativa Unión para la innovación; proyectos como Europa 2030 y procesos como el Proceso de Liubliana que permiten identificar y abordar los cambios necesarios tanto en las políticas productivas, como en las de investigación básica y aplicada. Si ya las evaluaciones intermedias de los programas de financiación como el 7 PM y el PIC fundamentan algunas reformas y simplificaciones iniciales, será a medida de que se complete el actual periodo de reflexión estratégica, cuando continuarán las reformas del derecho comunitario de diseño, financiación, gestión y evaluación del $\mathrm{I}+\mathrm{D}+\mathrm{i}$.

Pero, cuando hablamos de $\mathrm{I}+\mathrm{D}+\mathrm{i}$ como motor del desarrollo, no sólo aludimos a un conjunto de procesos cuyo desarrollo corresponde a científicos, tecnólogos, inventores e industriales que actúan de forma independiente, aleatoria o desorganizada. Al contrario, nos referimos 
actividades que reciben financiación, apoyo y estructura a través de instrumentos jurídicos: legislativos, administrativos, contractuales, etc.. Por eso, si el trinomio $\mathrm{I}+\mathrm{D}+\mathrm{i}$ es un motor para la recuperación, el Derecho debe ser el volante que los guíe. Esa parece ser la línea que se está siguiendo en la Unión Europea.

\section{PRINCIPAL BIBLIOGRAFÍA Y DOCUMENTACIÓN CONSULTADA}

Aно, E. et al., "Informe Aho, Crear una Europa innovadora", informe del grupo de expertos independientes europeos sobre I+D, presidente: seññor Esko Aho, enero de 2006.

\section{Comisión Europea}

Libro Verde "Del reto a la oportunidad: hacia un marco estratégico común para la financiación de la investigación y la innovación por la UE”, $\operatorname{COM}(2011) 48$ final.

Comunicación "Europa 2020: Una estrategia para un crecimiento inteligente, sostenible e integrador” COM(2010) 2020.

Propuesta de Reglamento del Parlamento Europeo y del Consejo sobre las normas financieras aplicables al presupuesto anual de la Unión $\operatorname{COM}(2010) 815$.

Comunicación "Iniciativa emblemática de Europa 2020 Unión por la innovación”-COM(2010) 546.

Simplificar la ejecución de los programas marco de investigación» $\operatorname{COM}(2010) 187$.

Commission Staff Working Document, European Competitiveness Report 2010 Accompanying document to the Communication from the Commission to the European Parliament, the Council, the European Economic and Social Committee and the Commette of the Regions. An integrated Industrial Policy for the Globalisation Era Putting Competitiveness and Sustainability at Front Stage $\{\mathrm{COM}(2010) 614\}\{\mathrm{SEC}(2010) 1272\}$ SEC(2010) 1276 final. 
"An Integrated Industrial Policy for the Globalisation Era Putting Competitiveness and Sustainability at Centre Stage" $\{\operatorname{SEC}(2010) 1272\}$, $\{\operatorname{SEC}(2010) 1276\}$.

Comunicación "Preparar nuestro futuro: desarrollo de una estrategia común en la UE para las tecnologías facilitadoras esenciales" $\operatorname{COM}(2009) 512$.

Marco temporal comunitario aplicable a las medidas de ayuda estatal para facilitar el acceso a la financiación en el actual contexto de crisis económica y financiera. Diario Oficial, núm. C 016 de 22/01/2009, p. 0001-0009.

Recomendación de la Comisión del 10 de abril de 2008 sobre la gestión de la propiedad intelectual en las actividades de transferencia de conocimientos y Código de buenas prácticas para las universidades y otros organismos públicos de investigación [C(2008) 1329 final.

Comunicación "Informe de aplicación del Programa Comunitario de Lisboa 2010” COM (2008) 881 final, Bruselas, 16 de diciembre de 2008.

Comunicación "Hacia una programación conjunta en investigación: trabajar juntos para abordar de forma más eficaz las cuestiones preocupantes comunes $\{$ SEC(2008) 2281\} \{SEC(2008) 2282\}” COM (2008)0468 final.

Propuesta de Reglamento, "Propuesta de reglamento del Consejo relativo al marco jurídico comunitario para las infraestructuras de investigación europeas (ERI)”, COM (2008) 467.

Comunicación, "Mejorar el sistema de patentes en Europa" (COM (2007) 165 final).

\section{Comité Económico y Social Europeo}

Dictamen del Comité Económico y Social Europeo (CES) sobre la "Comunicación de la Comisión al Consejo, al Parlamento Europeo, al Comité Económico y Social Europeo y al Comité de las Regiones Eliminación de obstáculos para las inversiones transfronterizas mediante fondos de capital riesgo" INT / 404 Inversiones transfronterizas, Bruselas, 22 de octubre de 2008.

Dictamen sobre la "Comunicación de la Comisión al Parlamento Europeo, al Consejo, al Comité Económico y Social Europeo y al Comité 
de las Regiones-Simplificar la ejecución de los programas marco de investigación $\operatorname{COM}(2010) 187$ final”.

Dictamen "Sobre la Comunicación de la Comisión Regiones europeas competitivas gracias a la investigación y la innovación-Una contribución para un mayor crecimiento y más y mejores puestos de trabajo COM(2007) 474 final”, DOUE C 211 de 2008”.

\section{Consejo Europeo}

Conclusiones del Consejo Europeo, febrero de 2011.

Conclusiones del Consejo Europeo, mayo de 2008.

Fernández-NovoA, C. et al., Manual de la propiedad industrial, Madrid, Barcelona, Buenos Aires, 2009.

ENGLAND, P., "Towards a single, pan-European standard: common concepts in UK and "continental European" patent law", European Intellectual Property Review, vol. 32, núm. 6, 2010.

ERAB, "Preparar a Europa para un nuevo Renacimiento: una visión estratégica del Espacio Europeo de Investigación”, Bruselas, 2009.

Grupo de Reflexión sobre el Futuro de la UE, Proyecto Europa 2030, Retos y oportunidades, Informe al Consejo Europeo del Grupo de Reflexión sobre el futuro de la UE en 2030, Luxemburgo, 2010.

Guedou, "Le système de brevet en Europe", tresor-eco, núm. 9, enero 2007.

Jaeger, Th., “The EU patent : cui bono et quo vadit?”, Common Market Law Review, vol. 47, núm. 1, febrero de 2010.

Kamar, E., "Beyond Competition for Incorporations", Georgetown Law Journal, augosto de 2006.

Lüder, T., "Legislative and Policy Developments in the European Union", Fordham, abril de 2005.

Muñoz de Bustillo, R. y Bonete Perales, R., Introducción a la Unión Europea: un análisis desde la economía, Madrid, 2009.

Pérez Carrillo, E. F., "Europa 2008: retos estratégicos y reforma”, Boletín Mexicano de Derecho Comparado, México, 2008.

Rohrbeck, R. y Pirelli, L., The European Institute of Innovation and Technology: How to Steer a Multi-Stakeholder Innovation Ecosystem (April 18, 
2010). DIME Conference-Organizing for Networked Innovation, April 2010. Available at SSRN: http: / /ssrn.com/abstract $=1591751$.

Tamames, R., ¿Cuándo y cómo acabará la crisis? Tratactus lógicus economicus, Madrid, Turpial, 2011.

William, C. et al., Intellectual Property: Patents, Copyright, Trademarks and Allied Rights, 7a. ed., Londres, Sweet \& Maxwell, 2010. 